\title{
A Real-Time Interactive Previsualization Platform for Motion Control System In Virtual Film Making
}

\author{
Wang Chunshui ${ }^{1,}$, Zhao Jianjun ${ }^{1, b}$ and Guo Yunhui ${ }^{1, c}$ \\ ${ }^{1}$ Building C, Beijing Film Academy, No. 4, Xitucheng Street, Haidian District, Beijing, China \\ achunshui.wang@gmail.com, bzhaojianjun@bfa.edu.cn, cguoyunhui@bfa.edu.cn
}

\begin{abstract}
Keywords: Virtual Film Making, Motion Control System, Real-Time Interactive Previz, Simulation System

Abstract. Motion control system is an irreplaceable technology for bringing creative ideas into reality in visual effects production. It could implement complex camera movements as well as repeat the same movement several times, but there still exists limitations for its expensiveness and complexity to operate. In on-set shooting, it often takes times of motion planning and shooting test to get desired footage, which is time consuming, low efficiency and high costs. If the shot could be previsualized at virtual environment in advance, it would save shooting time, raise efficiency and reduce the costs. By using this approach, all kinds of design and test work is pre-finished during the Previz stage.The motion data of the camera is able to be converted into formats supported by motion control system thus could be used on-set directly. To meet this requirement, this paper designs a real-time interactive Previz simulation platform which could be applied on motion control system. This system previsualizes footages and perform device testing in virtual environment, and the Previz data could be used directly on real motion control system in on-set shooting.
\end{abstract}

\section{Introduction}

Motion control system is a kind of mechanical system which use computer to precisely control electrical motor, which could make the mechanical components mobilized by motors moved accurately as expected with planned position and speed etc. In film production, this system is mainly used to get precise control of the camera movements. The accurate camera motion tracks are recorded and saved in computer with feature of repeatability and controllability.

There are mainly two advantages of motion control system. First, it is able to implement high complexity motion tracks which are impossible with regular shooting facilities. Second, with the capacity of repeating the same programmed camera movements precisely several times, high creative camera scenes could be achieved by compositing the multiple footages with exact same movements in visual effects production. Therefore, motion control systems play an important role in visual effects making.

Motion control system first appeared in 1970's. Famous director George Lucas shot some scenes for 'Star Wars' in 1977, which requires to compose the separately taken foreground and background footages samlessly. But by traditional means, there's no way to repeat a complex camera movement precisely. The research team led by John Dykstra, the visual effects supervisor, developed a motion control system. What they built is the first generation of motion control system controlled by computer, aiming for controlling camera movements. This device provides the functionality of recording and saving complex camera motion tracks, which could drive the camera shootings multiple times with the same camera movement. The definition of motion control system given by John Dykstra is, an interactive camera system which could synchronous control the motion tracks for cameras, subjects, lights and etc. and the speed of films move in camera simultaneously. Since then, motion control system was continuously improved by the company, and generations of devices which are more sophisticated and convenient were produced. At the moment, the mainstream is occupied by two motion control system suppliers, General Lift in US and Mark Roberts in UK. Their products are widely used in production of Hollywood blockbusters. 
The motion control system this paper studied is Milo from Mark Roberts Motion Control (MRMC) company. It is the most popular motion control system and an Oscars winner at the Scientific and Technical Awards, which is illustrated by Fig.1.

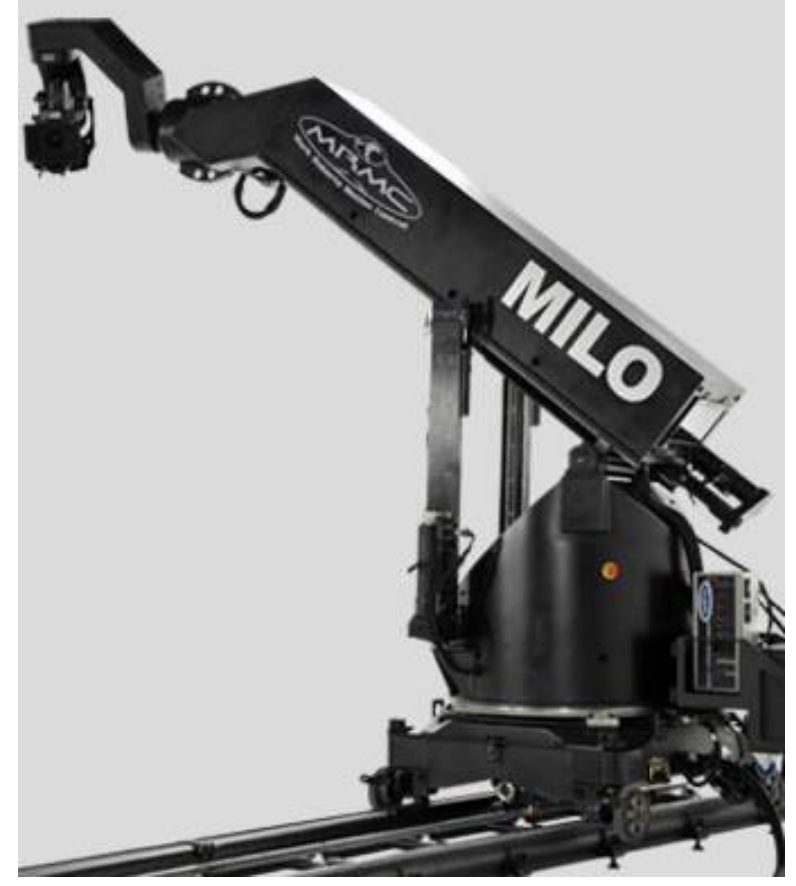

Fig.1 Milo from MRMC

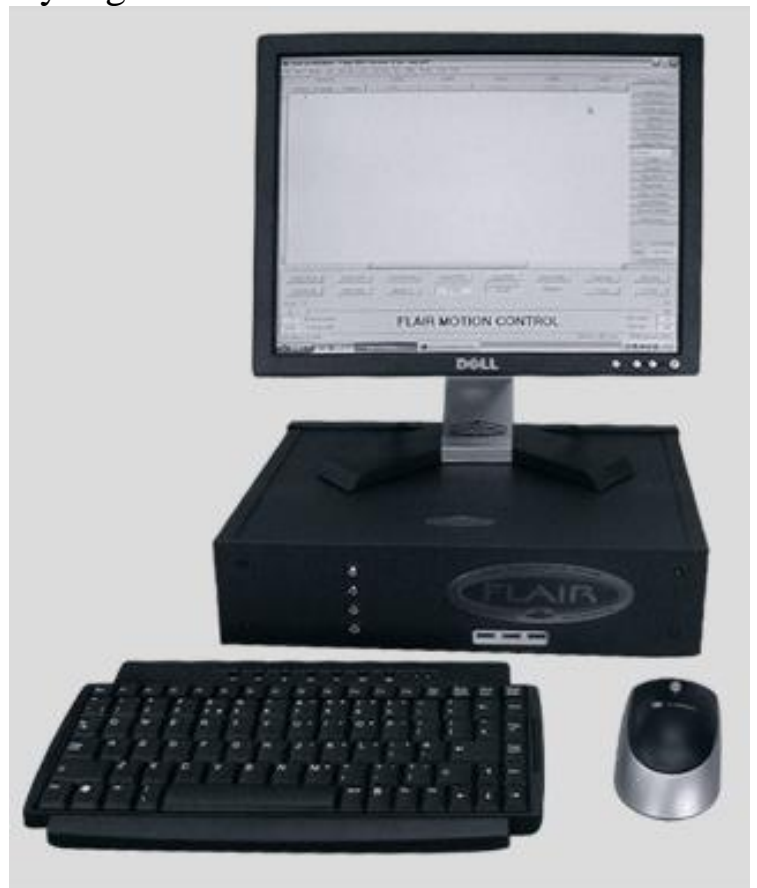

Fig.2 Flair, the operation software for Milo

\section{Working Principle of Motion Control System}

Motion control system is mainly comprised of computer control system, electrical control system and servo motor control system. First, the pulse data was transmitted by computer control system, which will be received later by electrical control system. Then, the electrical control system will power the servo motor system and produce a switch signal. After the pulse data and switch signal was detected by the servo motor system, the system will drive the corresponding conveyor belt or gear to carry out instructed action. The same camera motion tracks recorded by computer multiple times, is essentially numerous copies of the computer system instructions. As long as the pulse data transmitted by computer control system remains unchanged, the motion conveyed to the motors in the end will be the same. Thus the same camera motion track could be repeat precisely multiple times.

Motion control system drives the camera through different axes. Take Milo as an example, the 11 control axes include: Track, Rotate, Lift, Extend, Angle, Tilt, Pan, Roll, Focus, Zoom and Iris. These axes is the basic units used to control motion axes and lens parameters. The various data could be set and edited in the motion control system operation software. Fig.2 shows Flair, the motion control system operation software for Milo. The data for axes could be set in Flair, and the desired camera motion tracks could be achieved by the combination of axes data, which is shown by Fig3.

\begin{tabular}{|c|c|c|c|c|c|c|c|c|c|c|c|c|}
\hline \multirow{8}{*}{ 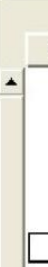 } & & & & 0.000 & 0.000 & 0.000 & 0.000 & 0.000 & 0.000 & 0.000 & 0.000 & 100000.00 \\
\hline & Frame & |Posns| & Target & Track & Lift & Rotate & Extend & Angle & Pan & Tilt & Roll & Focus \\
\hline & 0 & 1 & 26.16 & 568.669 & 4.256 & 67.256 & 52.238 & 33.825 & 89.579 & -43.072 & -46.329 & 26.16 \\
\hline & 113 & 2 & 48.00 & 542.445 & 8.005 & 57.000 & 51.456 & 45.000 & 32.520 & -21.210 & -37.963 & 48.00 \\
\hline & 147 & 3 & 51.84 & 507.569 & 7.589 & 23.235 & 45.250 & 27.449 & 74.764 & -30.711 & -34.193 & 51.84 \\
\hline & 198 & 4 & 70.13 & 497.856 & 11.236 & 20.560 & 40.137 & 22.321 & 91.754 & -45.060 & -43.490 & 70.13 \\
\hline & 214 & 5 & 76.46 & 490.256 & 6.257 & 54.000 & 39.639 & 20.173 & 95.989 & -40.603 & -41.060 & 76.46 \\
\hline & 2561 & 6 & 242.62 & 447.366 & 5.555 & 52.267 & 35.223 & 19.336 & 88.199 & 28.207 & -28.054 & 242.62 \\
\hline
\end{tabular}

Fig.3 Motion data of axes in motion control software

Motion control system is quiet expensive and complex for operation, the desired camera movements could be only achieved by the coordination of motion data of multiple axes. These 
complexity leads to several attempts of motion plans to finally get desired footages. This approach costs large number of valuable time and the efficiency on-set is pretty low. If the shots could be previsualized at virtual environment in advance, shooting time would be saved, efficiency would be raised and the cost would be reduced. Because by this approach, all kinds of design and test work is pre-finished during the Previz stage. The motion data of camera could be converted into formats supported by motion control system thus could be used on-set directly.

\section{Pre-existing Motion Control Previsualization Scheme}

Because of the complexity of adjusting and operating motion control system, MRMC company provided some schemes which could assist to test and debug the use of motion control system before the live action shooting. Two of pre-existing schemes are introduced below:

Use IKTRIX, A plug-in of Maya, to Proceed Previsualization of Milo Provided by MRMC Company. IKTrix could help to finish the previsualization of camera movements before the on-set shooting. It uses the virtual model of motion control system to exam if the movements is available in real space for on-set shooting. It would confirm if the camera movements are within the range of space and speed limits of motion control system. The configuration file used in software of real motion control system could be used directly to initialize the parameters of 3D virtual model of motion control system. IKTrix could solve for axes motion tracks of motion control system through the input camera motion track by inverse kinematic computing, and it also supports to exchange movements data between the model of motion control system within Maya and real motion control system.

The using steps of the plug-in is simply described below:

1 Proceed the shooting Previz of camera movements for shot scene.

2 Import the model of motion control system, place it at a proper position in scene, and set it to follow the camera movements.

3 Using motion control system in on-set shooting.

Use MC TOOLS Provided by MRMC Company to Implement the Data Transport between Milo and CGI Software and between CGI Softwares. MC Tools is an application software runs on Windows platform independently, it is mainly used to convert file data between common 3D softwares. There's a great many 3D softwares used to produce films and videos, which have its own file format and coordinate system preference. Different 3D softwares could be connected seamlessly through MC Tools, and the data transport between Milo and CGI software and between CGI softwares could be done easily.

MC Tools is not specifically developed for previsualization, but it is able to convert motion tracks set in 3D software to data files supported by real motion control system. It also has the ability to convert movement data from one 3D software to another, without the need to write any scripts, plug-ins or dirvers, and the data exchange from motion control to 3D software is also supported. For the moment, MC Tools support most of common 3D software such as Maya, 3DS Max, Softimage, Motion Builder, Cinema 4D, Houdini, Alias, Flame, Kuper and etc. MC Tools integrated the function to view and edit motion data, display the data in graph, scale and rotate motion track data for better display and etc. It could control the axes data of motion control system and even change the speed feature of movements and etc.

\section{The Architecture Design of General Usage Platform for Virtual Previsualization}

Overview of System Design. There exist some limits in both previsualization schemes for motion control system described above. Scheme 1 the IKTrix is a plug-in for software Maya, which is restricted to specific platform. And it only support animation previsualization for actual shooting, which is lack of real-time interactive operating and display for previsualization footage. Scheme 2 the MC Tools software only has the functionality of convert motion data between motion control system 
and common 3D softwares. The concrete functionality of motion planning, real-time interactive and display for previsualization footage need to be built and implemented by other systems.

According to the actual requirements and based on the advantages and disadvantages of two schemes described above, this paper designed and implemented a real-time interactive previsualization platform for virtual film making, which could be applied to multiple shooting devices including motion control system. The shooting test for virtual previsualization before the actual shooting could be implemented through this platform, and the virtual previsualization could be used as a means to instruct the actual shooting on-set. By this way, the target of saving time and money costs on-set could be achieved.

Overall Architecture Design. The virtual Previz platform is designed as a general platform which could be applied to virtual film making. The main function of this platform is to Previz shot before actual shooting on-set and test different shooting schemes and devices. The creative explorations and rehearsals for real shot could be finished in lab environment through this platform. The footages captured from Previz could be processed and edited for future saving and outputting. The camera movement data could be converted into data format supported by shooting device, which could be used directly on physical device on-set. And the final Previz footages could be also used as shooting instruction for different working departments on-set.

The top level of architecture design for virtual film making Previz platform mainly include several modules as below: real-time interactive control module, virtual film making Previz platform software core module, data exchange and real-time rendering output module etc. The virtual film making Previz platform software module is the core of virtual film making platform. The main function of this module is to process and convert the data for previsualization footage and Previz data for shots. This module is able to receive data input from real-time interactive control module, in addition to standard input method including mouse and keyboard, it also supports input from motion sensing device or virtual shooting system. This module uses real-time rendering output as direct reference for actual shooting, and the finished Previz footages and camera data is the final outcome for previsualization. The top level of architecture design for virtual film making Previz platform is shown by Fig.4.

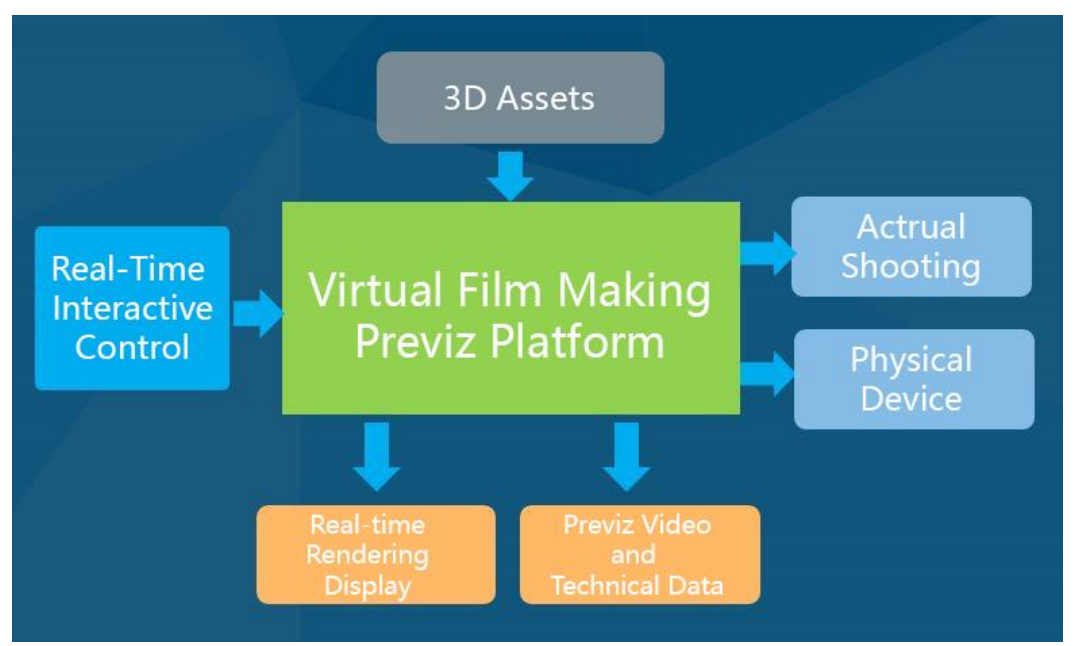

Fig.4 The top level of architecture design for virtual film making Previz platform

The modules and functionalities for this virtual Previz platform is introduced below:

Real-time interactive control module: as the camera motion control part for Previz, it is mainly in charge of the real-time interaction between virtual camera and virtual scene in virtual environment. This module is the external input module for virtual Previz platform, it provides a way with better availability and interactivity to finish the previsualization in virtual environments.

3D assets module: this module mainly provides some 3D assets to fast construction of virtual environment for virtual Previz platform. This module includes the modeling for actual shooting environments, rigging and animating for virtual characters, the virtualization for shooting devices including cameras and lenses etc. Through this module, the fast construction of virtual environments, character animations and camera movements becomes possible. 
Virtual film making Previz platform: this module is the core module of virtual film making Previz platform, most of core functionalities is implemented through this core. It also serves as data exchange center for previsualization data, receiving input data from real-time interactive module and outputing data files for previsualization footages and technical parameters.

Actual shooting and device module: the data converted from Previz platform used directly in actual shooting, which needs to finish the file convert in Previz platform between Previz data and files supported by physical devices.

Real-time rendering, Previz footages and technology data: using external display devices to display the rendering output generated from virtual camera in virtual environment, the edited output video could be used as final Previz sequences.

\section{Conclusion}

The cost is pretty high both in time and money when using motion control system due to its complexity in operation. With the aid of motion control previsualization platform designed and implemented by this paper, it would be possible to pre-test footage multiple times with low costs in lab environment. Once the desired motion data is obtained it could be used directly for on-set shooting, which can significantly reduce the time of on-set scheduling and device adjusting. Through this approach, enormous production costs could be spared thus this system has practical application value.

\section{Acknowledgements}

This work was financially supported by the Beijing Municipal Education Commission science and technology planning project(KM20140050002), and the research project of the State Administration of Press, Publication, Radio, Film and Television of The People's Republic of China(2015-53).

\section{References}

[1] Jingjie He, Su Wang, in: Advanced Motion Picture Technology, Vol. 06 (2015), p.45-48. In Chinese.

[2] Jin Liu, in: Journal of Beijing Film Academy, Vol. 02 (2004), p.51-61. In Chinese

[3] Jin Liu, in: Advanced Motion Picture Technology, Vol. 05 (2004), p.7-11. In Chinese

[4] Fei Qiu: Application Research of Camera Motion Control System, Beijing Film Academy. (2006) . In Chinese

[5] Information on http://www.mrmoco.com/cranes-rigs/products/motion-control-3d/ 